\title{
Screening traumatic life events in preschool aged children: cultural adaptation of Child and Adolescent Trauma Screen (CATS) Caregiver-report 3-6 years version
}

\author{
Pınar Zengin Akkuş ${ }^{1 \oplus}$, Esra Serdaroğlu² ${ }^{2 \oplus}$, Ayça Kömürlüoğlu ${ }^{3 \oplus}$, \\ Muhammed Asena ${ }^{4 \oplus}$, Evin İlter Bahadur ${ }^{1 \oplus}$, Gökçe Özdemir ${ }^{1 \oplus}$, \\ Sevilay Karahan ${ }^{5 \oplus}$, Elif N. Özmert ${ }^{1 \oplus}$ \\ Departments of ${ }^{1}$ Developmental Pediatrics and ${ }^{5}$ Biostatistics, Hacettepe University Faculty of Medicine, Ankara; ${ }^{2}$ Division of Pediatric \\ Neurology, Department of Pediatrics, Tokat State Hospital, Tokat; ${ }^{3}$ Department of Pediatrics, Cumhuriyet University Faculty of \\ Medicine, Sivas; 'Department of Pediatrics, University of Health Science, Gazi Yasargil Training and Research Hospital, Diyarbakur, \\ Turkey.
}

\begin{abstract}
Background. Given the high prevalence of potentially traumatic events (PTEs), pediatric providers are in a novel position in early identification and referral of the children with PTEs. Yet paucity of culturally adapted instruments to screen PTEs and related symptoms in preschool-aged children limits pediatric providers. This multicenter study aimed to screen the traumatic life events of preschool-aged children admitted to pediatric outpatient clinics at four different cities of Turkey and to determine the socio-demographic risk factors associated with PTEs. Moreover, it was also intended to develop the cultural adaptation of the Child and Adolescent Trauma Screen (CATS) Caregiver-report 3-6 Years version to Turkish and to analyze its reliability.
\end{abstract}

Methods. Participants of this multicenter study were collected from four different hospitals in three different regions (Central Anatolia, Black Sea, and Southeastern Anatolia regions) of Turkey. All parents of children at the age of 3-6 years who were admitted to the hospital for a clinical visit (sick-child or well-child visits) were invited to the study. In total, 188 preschool-aged children were included in the study. Socio-demographic characteristics of the child and the family were questioned and parents were asked to fill out the Turkish version of the CATS Caregiver-report.

Results. Internal consistency (Cronbach's alpha) of the Turkish version of the CATS Caregiver-report 3-6 Years was found as 0.86 . This study revealed that half of the participating children had experienced at least one PTE and more than one fourth experienced more than one event. Strikingly, no association between sociodemographic risk factors and PTE exposure was identified suggesting that PTE exposure is indeed widespread in our study population.

Conclusion. The findings of this study manifest the importance of routine screening of PTEs and related symptoms in children.

Key words: Potentially traumatic events, trauma screening, early childhood, cultural adaptation.

Children may encounter various kinds of possible traumatic events throughout their lives. Based on World Health Organization surveys, childhood adversities were reported

• Pınar Zengin Akkuş

pinarzenginakkus@gmail.com

Received 8th June 2020, revised 6th July 2020, accepted 22nd July 2020. by more than one-third of the population. ${ }^{1}$ These adverse experiences can be perceived as positive, tolerable, or toxic stress depending on the intensity, duration, timing of the event, and the support given by caregivers. ${ }^{2}$ Sustained adversities perceived as toxic stressors were reported to yield various longterm consequences on child's health and development. ${ }^{3,4}$ However, some of these events 
are preventable and their unfavorable effects can be improved by activating supporting systems and early interventions.

Given the high prevalence of potentially traumatic events (PTEs) and its impact on health, the concept of trauma-informed care has gained importance. Trauma-informed care involves understanding, recognizing, and responding to the impacts of all kinds of trauma. Routinely screening for trauma exposure and related symptoms is the first step of trauma-informed care. ${ }^{5}$ Moreover, pediatric providers are in a novel position to identify the children and families with at risk of adverse experiences. ${ }^{6}$ American Academy of Pediatrics (AAP) issued a technical report including recommendations for pediatricians to screen children and their families who are at risk for toxic stress. ${ }^{7}$

Screening with standardized tools is needed for better recognition of traumatic experiences in the lives of children and the impact of events on their functioning. In the literature, there are many available instruments for screening and assessment of trauma in children and adolescents. ${ }^{8}$ However, there are few instruments to screen PTEs and posttraumatic stress symptoms (PTSS) of preschool-aged children. ${ }^{9}$

Considering the recommendations of AAP, this study intended to screen the traumatic life events of preschool-aged children admitted to pediatric outpatient clinics at four different cities of Turkey in three different regions. In addition, it was aimed to define the sociodemographic risk factors associated with potentially traumatic events. Due to the paucity of validated screening instruments in the Turkish language for preschool-aged children, it was also intended to develop the cultural adaptation of the Child and Adolescent Trauma Screen (CATS) Caregiver-report 3-6 Years version to Turkish and to determine its reliability. The CATS questionnaire, which is a screening instrument based on the DSM- 5 criteria for Posttraumatic Stress Disorder, is developed to measure PTEs and PTSS. The
CATS Caregiver-report 3-6 Years version is used in preschool-aged children based on caregiver report.

\section{Material and Methods}

\section{Study population}

This multicenter study was designed by the Department of Developmental Pediatrics at Hacettepe University Faculty of Medicine. Participants of this study were collected from four different hospitals (Hacettepe University Hospital, Sivas Cumhuriyet University Hospital, Tokat State Hospital, and Diyarbakir Gazi Yasargil Training and Research Hospital) in three different regions (Central Anatolia, Black Sea, and Southeastern Anatolia regions) of Turkey. The sample size for the reliability study was determined to be at least 150 (10 for each item of CATS) participants. Therefore, it was planned for each four investigators from each hospital to include 50 parents to the study. The data was collected between April-November 2019. All parents of children at the age of 3-6 years who were admitted to the hospital for a clinical visit (sick-child or well-child visits) with various reasons were invited to the study. Parents who accepted to participate were included in the study. Approval for the research project was obtained from the Hacettepe University Ethics Committee (GO 19/388). Informed consent was obtained from all individual participants included in the study.

\section{Procedures}

In the first part of the interview, demographic characteristics of the child and the family (age, gender of the child, additional chronic illnesses of the child, educational level, occupation, and socioeconomic status of the family, determined by the Hollingshead-Redich index ${ }^{10}$ ) were questioned. Based on the Hollingshead-Redich index, parents were divided into three groups as low, middle, and high.

In the second part, parents were asked to fill out the Turkish version of the CATS Caregiver- 
report. The CATS is a questionnaire to screen for exposure to PTEs and PTSS. ${ }^{11}$ The CATS Caregiver-report 3-6 Years has a 15-item structured PTEs checklist and if at least one PTE is evident, parents are asked to fill out the 16-item PTSS checklist with a rating scale of $0=$ never, $1=$ once in a while, $2=$ half the time, and $3=$ almost always. The PTEs checklist includes items on natural disasters (serious natural disaster like a flood, tornado, hurricane, earthquake, or fire), accidents (serious accident or injury like a car/ bike crash, dog bite, sports injury), experiencing or seeing violence (to be slapped, punched, or beat up/seeing someone get slapped, punched, or beat up), sexual abuse (to be touched private parts), traumatic loss (sudden or violent death of someone close to child), medical procedures (stressful or scary medical procedure) and wars (being exposed to wars). The CATS is based on the DSM- 5 criteria and items map onto criteria $\mathrm{B}$ : intrusions (having bad dreams related to a stressful event, etc.), C: avoidance (avoiding anything that is a reminder of a stressful event, etc.) D: negative alterations in cognitions and mood (increase in negative emotional states, etc.) and E: hyperarousal (being overly alert or on guard, etc.). Lastly, CATS involves questions on the interference of PTSS in five key areas of functioning (getting along with others, school, hobbies, family relationships, and general happiness). ${ }^{11}$ It is worth noting that since the CATS questionnaire is developed as a screening instrument, the clinical symptoms should be diagnosed by the clinical interviews of mental health professionals. In United States sample, Cronbach's alpha coefficient of preschool version was reported as $0.92 .{ }^{11}$

CATS is a license-free open access instrument. ${ }^{12}$ Permission was granted from the first author of the international CATS study ${ }^{11}$ for the translation and cultural adaptation of the original Caregiver-report 3-6 Years. The CATS Caregiver-report 3-6 Years was first translated from English to Turkish by two of the authors, resulting in a single version after the consensus meeting. Then an independent native speaker back translated the scale into English. After a pilot study with five patients, minor adaptations were made for cultural suitability and the final Turkish version of the CATS Caregiver-report 3-6 Years was obtained.

The researchers and clinicians can reach the Turkish version of the CATS Caregiver-report 3-6 Years at this link ${ }^{12}$ and cite the current article.

\section{Statistical analysis}

Statistical analyses were performed utilizing the IBM SPSS for Windows Version 22.0. Numerical variables were summarized as mean \pm standard deviation or median $\left[25^{\text {th }}-75^{\text {th }}\right.$ percentile]. Categorical variables were given as frequencies and percentages. Categorical variables were compared by chi square test. Normality of the continuous variables was evaluated by Kolmogorov-Smirnov test. Homogeneity of variances was tested by Levene test. Differences between the groups according to continuous variables were determined by independent samples $\mathrm{t}$ test or Mann-Whitney $\mathrm{U}$ test as appropriate. One-way ANOVA or KruskalWallis test was used to compare more than two independent groups. Post hoc comparisons were done by Tukey HSD or Dunn test. Relation between continuous variables was determined by Spearman correlation coefficient. Internal consistency of scale was given by Cronbach's alpha coefficient. A p value less than 0.05 was considered as significant.

\section{Results}

Parents of 188 children were enrolled. The numbers of the participants from four hospitals were 53,50, 35, and 50 respectively. Demographic features of the participants are presented in Table I.

Internal consistency (Cronbach's alpha) of the Turkish version of the CATS Caregiverreport 3-6 Years was found as 0.86. Internal consistencies of four clusters are given in Table II.

According to the CATS questionnaire, 49.5\% of the study population experienced at least 
Table I. Demographic features of participants.

\begin{tabular}{|c|c|}
\hline Demographic features & Results (N: 188) \\
\hline Male/ female, $\mathrm{n} / \mathrm{n}$ & $103 / 85$ \\
\hline Age of child (years) ${ }^{\mathrm{a}}$ & $4.5[3-6]$ \\
\hline \multicolumn{2}{|l|}{ Chronic health condition of child, $\mathrm{n}(\%)$} \\
\hline None & $143(76.1)$ \\
\hline Neurodevelopmental disorder & $35(18.6)$ \\
\hline Other chronic diseases & $10(5.3)$ \\
\hline Age of mother (years) ${ }^{b}$ & $33.2 \pm 5.7$ \\
\hline Age of father (years) ${ }^{b}$ & $36.9 \pm 6.1$ \\
\hline Total education duration of mother (years) ${ }^{b}$ & $9.3 \pm 4.8$ \\
\hline Total education duration of father (years) ${ }^{\mathrm{b}}$ & $10.5 \pm 4.5$ \\
\hline \multicolumn{2}{|l|}{ Socioeconomic status (Hollingshead index), n (\%) } \\
\hline High & $49(26.1)$ \\
\hline Middle & $35(18.6)$ \\
\hline Low & $104(55.3)$ \\
\hline Lower income (<2000 Turkish Lira/per month), n (\%) & $55(29.3)$ \\
\hline Preschool enrollment, $\mathrm{n}(\%)$ & $91(48.4)$ \\
\hline
\end{tabular}

a: Median value and minimum -maximum values are presented.

b: Mean value and standard deviation are presented.

Table II. Internal consistency of the Turkish version of CATS Caregiver-report 3-6 years.

\begin{tabular}{lc}
\hline & Cronbach's alpha \\
\hline CATS total score & 0.86 \\
Cluster B: Re-experiencing & 0.73 \\
Cluster C: Avoidance & 0.62 \\
Cluster D: Negative alterations in cognitions and mood & 0.62 \\
Cluster E: Hyperarousal & 0.74 \\
\hline
\end{tabular}

one of the potentially traumatic events. While $20.7 \%$ of participants were exposed to one PTE, 28.7\% were exposed to more than one PTE. The percentages of the participants who experienced at least one PTE were similar in Hacettepe University Hospital (56.6\%), Sivas Cumhuriyet University Hospital (57.1\%), and Tokat Satate Hospital (56.0\%). However, it was $30.0 \%$ in Diyarbakır Gazi Yaşargil Training and Research Hospital and this difference was statistically significant $(\mathrm{p}=0.016)$.

Among 15 PTEs, the most common events experienced by the children in the study population were: i) stressful or scary medical procedure $(23 \%)$, ii) slapped, punched, or beat up in their family $(16 \%)$, iii) seeing someone in the community get slapped, punched (14\%), and iv) seeing someone in the family get slapped, punched, or beat up (13\%) and another stressful or scary event $(13 \%)$ in the study population. Among 93 participants who experienced at least one PTE, 20 had scores of PTSS higher than the clinical cut-off $(\geq 16)$. The children with high scores of PTSS (10.6\%) in the study group were referred to mental health professionals.

Participants were divided into two groups: i) children without any PTEs and ii) children with at least one PTE. The comparisons of the demographic features of these two groups are presented in Table III. Between the two groups, gender, age of the child, diseases of the child, age of the parents, education durations of the 
Table III. Comparison of demographic features between children with and without PTEs.

\begin{tabular}{lccc}
\hline Demographic features & $\begin{array}{c}\text { Children without } \\
\text { PTEs (N: 95) }\end{array}$ & $\begin{array}{c}\text { Children with } \geq 1 \\
\text { PTEs (N: 93) }\end{array}$ & p value \\
\hline Child characteristics & & & \\
\hline Male gender, $\mathrm{n}(\%)$ & $51(53.7)$ & $52(55.9)$ & 0.759 \\
Age of child (years) $^{\mathrm{a}}$ & $4.4 \pm 1.0$ & $4.5 \pm 1.1$ & 0.879 \\
Presence of chronic disease, $\mathrm{n}(\%)$ & & & 0.145 \\
$\quad$ Developmental problems & $12(12.6)$ & $6(6.5)$ & \\
$\quad 10(10.5)$ & $17(18.2)$ & 0.146 \\
$\quad$ Other chronic disease & $41(43.1)$ & $50(53.7)$ & \\
Preschool enrollment, $\mathrm{n}(\%)^{\text {Family characteristics }}$ & & & 0.267 \\
\hline Age of mother & $32.7 \pm 5.9$ & $33.6(5.4)$ & 0.216 \\
Age of father & $36.4 \pm 6.2$ & $37.5(6.0)$ & 0.342 \\
Education of mother (years) $^{\mathrm{a}}$ & $9.0 \pm 4.9$ & $9.5(4.6)$ & 0.44 \\
Education of father (years) $^{\mathrm{a}}$ & $10.3 \pm 4.9$ & $10.7(4.2)$ & 0.827 \\
Number of children $^{\mathrm{b}}$ & $2[1-6]$ & $2[1-9]$ & 0.673 \\
Family size $^{\mathrm{b}}$ & $4[1-5]$ & $4[1-5]$ & 0.365 \\
Socioeconomic status $^{\mathrm{b}}$ & $4[3-10]$ & $4[3-10]$ & 0.398 \\
Household income $^{\mathrm{b}}$ & $2[1-4]$ & $2[1-4]$ & \\
\hline
\end{tabular}

a: Mean value and standard deviation are presented

${ }^{\mathrm{b}}$ : Median value and minimum-maximum values are presented.

parents, socioeconomic status and income of families, family size, and number of children were not statistically significantly different. Therefore, models to explore the factors to predict the presence of PTEs were not significant.

\section{Discussion}

Assessments of child trauma exposure and posttraumatic symptoms were reported to be challenging especially in preschool-aged children. ${ }^{13,14}$ Moreover, paucity of linguistically and culturally adapted screening instruments for young children could be a limiting factor for international health care providers. The general Cronbach's alpha coefficient of the Turkish version of the CATS Caregiver-report 3-6 Years was quite similar to that of the original study. Although, Cronbach's alpha coefficients of clusters of CATS Caregiver-report 3-6 Years were not provided in the original study, in the current study, they were found similar to the values of CATS Caregiver-report 7-17 Years. ${ }^{11}$ Since the results of this study demonstrated a good internal consistency of the Turkish version of the CATS Caregiver-report 3-6 Years, we recommend health care providers to utilize this instrument as a screening tool for PTEs and related PTSS in preschool-aged children.

This study revealed that almost half of the participating children have experienced at least one of the PTEs and more than one-fourth of the study population experienced more than one event. The proportion of PTEs exposure can be affected by the type of screening instrument and the type of setting that participants were recruited from. Due to the lack of studies using the same instrument to screen PTEs in hospital settings, we could not directly compare our findings with the literature. The proportion of one PTE exposure was higher than that of a previous study where the percentage of any childhood adversity was reported to be $38.9 \%$ in high-middle income countries. ${ }^{1}$ This dissimilarity could be linked to the abundance of PTEs type of 'stressful or scary medical procedure' experienced by our participants, which is understandable since we recruited them from hospital settings. However, there 
exist other studies reporting higher percentages of preschool-aged children with a history of at least one traumatic event. ${ }^{15,16}$ Moreover, a study reported that half of the preschool-aged children living in poverty have been exposed to one or more PTEs. ${ }^{17}$ Likewise, the majority of our participants belong to low socioeconomic status and we found half of the participants have experienced at least one of the PTEs. However, we did not find an association of socioeconomic status with either the presence or the number of traumatic events, in contrast to several previous reports. ${ }^{17,18}$

There have been inconsistent reports on the gender differences in PTE exposure. ${ }^{17,19}$ We found no differences between female and male participants in terms of exposure to PTEs. On the other hand, in the literature, there have been few studies with controversial finding $\mathrm{s}^{20-22}$ on the association between adverse childhood experiences and chronic conditions or developmental problems of preschool-aged children. In this study, PTEs exposure was not found to be more prevalent in children with developmental problems compared to typically developing children. Moreover, the prevalence of chronic conditions was not statistically different between children without PTEs, with one PTE and more than one PTE. In addition, we did not identify any other socio-demographic risk factor associated with PTEs exposure suggesting that experiencing PTEs was, in fact, widespread in our study population.

During the interpretation of the current results, limitations should not be disregarded. Since we recruited participants who were admitted to hospitals for various reasons, our PTEs rates may not be generalized to community settings. Another limitation is that the present study did not focus on coping mechanisms or resilience factors of children. On the other hand, due to the sample size, the factors underlying the lower PTEs percentage in one of the centers compared to the other three centers could not be identified. Finally, we culturally adapted and analyzed the reliability of the Turkish version of the CATS Caregiver-report 3-6 Years but we did not conduct a standardization study of the instrument. On the other hand, one may suspect the risk of underreporting of PTE exposure by caregivers due to desirability effects. However, we believe that this was not a limitation of the current study since the rates of PTEs were already higher than the previous reports. ${ }^{1}$

To the best of authors' knowledge, this multicenter study with a diverse population from four different hospitals in Turkey, is the first national and one of the several international studies screening the possible traumatic life events in preschool-aged children. This study revealed that a considerable proportion of children were exposed to PTEs and approximately $20 \%$ of them had high scores of PTSS. Given the high prevalence of PTEs, the findings manifested the importance of routine screening of PTEs and related symptoms in children.

The current study that screened PTE exposure and related symptoms among children who were admitted to hospitals with various reasons revealed a high prevalence of exposure to PTEs. Despite its high prevalence, pediatric providers donot routinely screen children for PTEexposure and possibly overlook the impact of exposure. Mental health professionals utilize multiple methods for the comprehensive assessment of trauma and diagnosis of post-traumatic stress disorders. ${ }^{14}$ However, our findings highlighted the need for screening PTE exposure and related symptoms by pediatricians who would have an important role in early identification of traumaexposed children and their referral to mental health professionals, thereby interventions can be developed to reduce exposure and its impact on children.

\section{REFERENCES}

1. Kessler RC, McLaughlin KA, Green JG, et al. Childhood adversities and adult psychopathology in the WHO World Mental Health Surveys. Br J Psychiatry 2010; 197: 378-385. 
2. National Scientific Council on the Developing Child. Excessive stress disrupts the development of brain architecture. J Child Serv 2014; 9: 143-153.

3. Shonkoff JP, Boyce WT, McEwen BS. Neuroscience, molecular biology, and the childhood roots of health disparities: building a new framework for health promotion and disease prevention. JAMA 2009; 301: 2252-2259.

4. Anda RF, Felitti VJ, Bremner JD, et al. The enduring effects of abuse and related adverse experiences in childhood. A convergence of evidence from neurobiology and epidemiology. Eur Arch Psychiatry Clin Neurosci 2006; 256: 174-186.

5. Center for Substance Abuse T. SAMHSA/CSAT Treatment Improvement Protocols. TraumaInformed Care in Behavioral Health Services. Rockville, MD: Substance Abuse and Mental Health Services Administration (US), 2014.

6. Cohen JA, Kelleher KJ, Mannarino AP. Identifying, treating, and referring traumatized children: the role of pediatric providers. Arch Pediatr Adolesc Med 2008; 162: 447-452.

7. Garner AS, Shonkoff JP. Committee on Psychosocial Aspects of Child and Family Health; Committee on Early Childhood, Adoption, and Dependent Care; Section on Developmental and Behavioral Pediatrics. Early childhood adversity, toxic stress, and the role of the pediatrician: translating developmental science into lifelong health. Pediatrics 2012; 129: e224-e231.

8. Eklund K, Rossen E, Koriakin T, Chafouleas SM, Resnick C. A systematic review of trauma screening measures for children and adolescents. Sch Psychol Q 2018; 33: 30-43.

9. Strand VC, Sarmiento TL, Pasquale LE. Assessment and screening tools for trauma in children and adolescents: a review. Trauma Violence Abuse 2005; 6: 55-78.

10. Hollingshead AB, Redlich FC. Social class and mental illness: a community study. 1958. Am J Public Health 2007; 97: 1756-1757.

11. Sachser C, Berliner L, Holt T, et al. International development and psychometric properties of the Child and Adolescent Trauma Screen (CATS). J Affect Disord 2017; 210: 189-195.
12. Ulmer Onlineklinik. Available at: https://ulmeronlineklinik.de/course/view.php?id=1701. (Accessed 30/03/2020).

13. Glackin E, Forbes D, Heberle A, Carter A, Gray SAO. Caregiver self-reports and reporting of their preschoolers' trauma exposure: discordance across assessment methods. Traumatology (Tallahass Fla) 2019; 25: 172-180.

14. Stover CS, Berkowitz S. Assessing violence exposure and trauma symptoms in young children: a critical review of measures. J Trauma Stress 2005; 18: 707717.

15. Grasso DJ, Ford JD, Briggs-Gowan MJ. Early life trauma exposure and stress sensitivity in young children. Pediatr Psychol 2013; 38: 94-103.

16. Roberts YH, Ferguson M, Crusto CA. Exposure to traumatic events and health-related quality of life in preschool-aged children. Qual Life Res 2013; 22: 2159-2168.

17. Briggs-Gowan MJ, Ford JD, Fraleigh L, McCarthy K, Carter AS. Prevalence of exposure to potentially traumatic events in a healthy birth cohort of very young children in the northeastern United States. J Trauma Stress 2010; 23: 725-733.

18. Bush HH, Eisenhower A. Exposure to interpersonal violence and socioemotional adjustment in economically disadvantaged preschoolers. J Genet Psychol 2014; 175: 214-232.

19. Tolin DF, Foa EB. Sex differences in trauma and posttraumatic stress disorder: a quantitative review of 25 years of research. Psychol Bull 2008; 132: 959992.

20. Burke NJ, Hellman JL, Scott BG, Weems CF, Carrion VG. The impact of adverse childhood experiences on an urban pediatric population. Child Abuse Negl 2011; 35: 408-413.

21. Marie-Mitchell A, O'Connor TG. Adverse childhood experiences: translating knowledge into identification of children at risk for poor outcomes. Acad Pediatr 2013; 13: 14-19.

22. von Sneidern E, Cabrera KP, Galeano N, Plaza M, Barrios M. Association between adverse childhood experiences (ACEs) and developmental delay of preschool children in a rural area of Colombia. J Child Adolesc Trauma 2017; 10: 225-232. 